\title{
Research on the Mechanism of T Cell Subsets and Cytokines in Hashimoto's Thyroiditis
}

\author{
Jing Feng ${ }^{1}$, Zhaoxin $\mathrm{Mu}^{2}$, Xinsheng $\mathrm{Li}^{1}$, Zhenjiang $\mathrm{Hou}^{2, *}$ \\ ${ }^{1}$ Cangzhou Central Hospital, Cangzhou, China \\ ${ }^{2}$ Institute of Thyroid Diseases Affiliated to Cangzhou Medical College, Cangzhou Thyroid Disease Engineering Technology Research Center, \\ Cangzhou, China
}

Email address:

houzhenjiang@sina.com (Zhenjiang Hou)

${ }^{*}$ Corresponding author

\section{To cite this article:}

Jing Feng, Zhaoxin Mu, Xinsheng Li, Zhenjiang Hou. Research on the Mechanism of T Cell Subsets and Cytokines in Hashimoto's Thyroiditis. American Journal of Biomedical and Life Sciences. Vol. 8, No. 4, 2020, pp. 83-90. doi: 10.11648/j.ajbls.20200804.14

Received: May 28, 2020; Accepted: July 10, 2020; Published: July 23, 2020

\begin{abstract}
As being one of the most common diseases in autoimmune thyroid disease (AITD), Hashimoto's thyroiditis (HT) is an organ-specific autoimmune disease. It is mostly related to genetics, infection, and excessive iodine, but the exact pathogenesis has not yet clear. As one of the most important immune cells, $\mathrm{T}$ cells play an important role in the human immune. Helper $\mathrm{T}$ cells (Th) and regulatory T cells (Treg) are two important subgroups of T cells. The former include Th1, Th2, Th17 and other cells. HT patients is mainly characterized by expressing Th1 cytokines. The imbalance of $\mathrm{Th} 1 / \mathrm{Th} 2$ ratio can induce abnormal immune response, which is closely related to the incidence of HT. Th17/Treg cells are mutually restricted in differentiation and mutually antagonistic in function. IL-17 secreted by Th17 cells directly promotes the inflammatory response of thyroid tissue and accelerates the damage of thyroid tissue. Abnormal Treg cell function cannot effectively inhibit the occurrence of autoimmune reactions and promote immune tolerance. Th17/Treg constitute a relatively independent group of cell networks except Th1/Th2. Under normal circumstances, Th1/Th2 and Th17/Treg cells maintain a dynamic balance. However, once unbalanced, they will lead to immune dysfunction and participate in the development of HT. This article reviews the mechanisms of Th1/Th2 and Th17/Treg cells and their cytokines in the pathogenesis of HT.
\end{abstract}

Keywords: Thl/Th2 Cells, Th17/Treg Cells, Retinoic Acid-related Orphan Receptors (ROR- $\gamma \mathrm{t}$ ), Forkhead Box p3(Foxp3), Hashimoto Thyroid Inflammation

\section{Introduction}

$\mathrm{T}$ cells that mainly included $\mathrm{CD} 4^{+} \mathrm{T}$ and $\mathrm{CD} 8^{+} \mathrm{T}$ cells are the most numerous and most complex type of cells in lymphocytes. It plays an important role in cellular immunity and assisted in humoral immune response [1]. T lymphocytes are classified into $\mathrm{CD} 4^{+}$helper $\mathrm{T}$ cells $(\mathrm{Th})$, regulatory $\mathrm{T}$ cells (Treg), effector $\mathrm{T}$ cells (effect $\mathrm{T}$ cells, Teff) and cytotoxic $\mathrm{T}$ lymphocyte (CTL), according to their different functions in the immune response, etc. After being mediated by $\mathrm{T}$ cell receptor (TCR) and induced by cytokines, $\mathrm{CD} 4{ }^{+} \mathrm{T}$ cells are mainly divided into four cell subpopulations: Th1, Th2, Th17 and Treg. They are mainly interact with and restrict to each other through secreting cytokines so as to jointly maintain the immune balance. Th1 and Th2 cells are the first discovered
$\mathrm{CD}^{+} \mathrm{T}$ cell subsets, and $\mathrm{Th} 1 / \mathrm{Th} 2$ imbalance is considered to be the main pathogenesis of autoimmune diseases (AID). In recent years, as two new subsets of $\mathrm{CD} 4^{+} \mathrm{T}$ cells, Th17 and Treg being different from Th1 and Th2 cells has become a hot research topic in the regulation of inflammatory response [2]. The research found that $90 \%$ of thyroid diseases were mainly manifested as autoimmune thyroid diseases (AITD) whose incidence had been on the rise in recent years, which was the most common thyroid disease except iodine deficiency disease and a common organ-specific AID in humans. AITD mainly include Hashimoto thyroiditis (HT) and diffuse toxic goiter (Graves' Disease, GD disease), which is characterized by hypothyroidism and hyperthyroidism, respectively. The main feature of HT is the formation of specific autoantibodies in the body, such as anti-thyroid peroxidase antibody (anti-thyroid peroxidase antibody, 
TPO-Ab), anti-thyroid globulin antibody (anti-thyroid globulin antibody, TG-Ab) and the infiltration of a large number of lymphocytes, leading to an abnormal number and function of organ-specific autoimmune cells and disrupting cellular immunity and humoral immunity. The research found that $\mathrm{T}$ Cell Subsets and cytokines were involved in the pathogenesis of HT and were closely related to the occurrence of disease. This article reviews the mechanism of Th1/Th2 and Th17/Treg cells and their cytokines in the pathogenesis of HT, so as to deepen the understanding of $\mathrm{T}$ cell subsets and cytokines in the pathogenesis of HT, broaden the research ideas of HT, thus opening up new ways to effectively develop the prevention and treatment of HT.

\section{Thl / Th2 Cells and Hashimoto's Thyroiditis}

Helper T cells play an important regulatory role in specific immune responses. Th1 and Th2 are two important subpopulations of activated antigen-specific $\mathrm{CD}^{+} \mathrm{T}$ cells. Th1 cells mainly secrete inflammatory cytokines such as interleukin 2 (interleukin, IL-2), interferons (interferons, IFNs) and so on, being aimed at inhibiting the function of $\mathrm{B}$ cells, mediating cellular immunity and playing an important role in the process of anti-infection and induction of AID Role [3]. Th2 cells mainly secrete IL-4, IL-10 and other cytokines, with the purpose of mainly mediating humoral immunity, assisting in the production of antibodies and playing a leading role in the process of anti-allergy and removal of parasites [4]. Th1 and Th2 cells interactively regulate through cytokines secreted by themselves or other immune cells to jointly maintain the dynamic balance of Th1/Th2 and cellular immunity and humoral immunity, and protect the body from damage. Once unbalanced, there will be a dominant response of Th1 or Th2 cells, triggering an abnormal immune response and leading to the occurrence of disease [5]. HT is a chronic inflammatory autoimmune thyroiditis (AIT) that uses its own thyroid tissue as an antigen also being known as lymphocytic thyroiditis. It is the most common clinical thyroid inflammation and the most common cause of clinical and subclinical hypothyroidism. In HT patients, there are thyroid autoantibodies and a large number of inflammatory cell infiltration and follicle destruction to varying degrees, which eventually lead to hypothyroidism, especially in middle-aged women. The incidence of HT is mostly related to environmental, genetic and immune disorders [6], but the pathogenesis has not yet clear. Most researches recognize that HT is related to the dominant population of Th1 cells and the types of secreting cytokines. IL-12 depending on Th1 is closely related to the pathogenesis of HT. Exogenous IL-2 can stimulate the body to secrete high levels of IFN- $\gamma$ and TNF- $\alpha$. The former can promote the occurrence of AIT. Karanikas et al. found for the first time that the expression of Th1 cytokines in HT patients was related to the TPO-Ab titer, and the secretion of TNF- $\alpha$ and IFN- $\gamma$ in patients with high antibody titers was significantly higher than that in the low titers and control. Experimental animal models of autoimmune thyroiditis (EAT) confirmed that when EAT developed into fibrosis, IFN- $\gamma$ was expressed at high levels, but there were reports of IFN- $\gamma$ expression early in EAT [7]. Research has shown that inflammatory factors secreted by Th1 cells are involved in the pathogenesis of HT. HT is a cellular immune response mediated by Th1. Th1 cells can activate CTL and macrophages, destroy thyroid follicular epithelial cells, and cause thyroid tissue damage and the occurrence of thyroiditis [8]. It is currently believed that Th1-type cytokine-mediated cellular immunity plays a major role in the pathogenesis of HT.

A study [9] found that both Th1 and Th2 cytokines were involved in the pathogenesis of HT. Th1 cytokines (IL-2, IFN- $\gamma$ ) and Th2 cytokines (IL-4, IL-10) could be detected in the early and peak periods of EAT, and the ratio of Th1/Th2 cytokines was basically similar at the beginning and peak of the diseaser, which indicated that both Th1 and Th2 cytokines were involved in the pathogenesis of EAT. Roura-Mir et al. found that the expression of Th1 cytokines was dominant in HT patients. Jiang Ling [10] believed that Th1 cytokines were involved in the mediated cellular immunity of HT patients and played a major role in the pathogenesis of HT. Serum IL-2, TNF- $\gamma$ and TNF- $\alpha$ in HT patients were significantly higher than that in the control group. After stimulation with concanavalin A, the levels of IL-2 and TNF-asecreted by peripheral blood lymphocytes also increased significantly, which indicated that Th1 cells Factors (IL-2, TNF- $\alpha$, IFN- $\gamma$ ) might be related to the pathogenesis of chronic lymphocytic thyroiditis. IFN- $\gamma$ could not only promote the infiltration of lymphocytes and occerance of AIT in the thyroid, but also promote the activation of infiltrating lymphocytes and macrophages and the release of cytokines such as IL-1, IL-6, TNF- $\alpha$, etc. The destruction of tissues suggested that IFN- $\gamma$ secreted by Th1 could promote the occurrence of AIT. Kotani et al. found by needle biopsy that the positive rate of DNA fragmentation of thyroid cells in lymphocyte infiltration sites of HT patients was high, and apoptotic bodies could be seen under electron microscopy. It was speculated that infiltrating lymphocytes might release cytokines such as TNF- $\alpha$ and TNF- $\beta$, which together with CTL and NK cells induced apoptosis of thyroid cells leading to tissue damage. Nanba et al. [11] reported that Th1 cells $\left(\mathrm{CD}^{+}{ }^{+} \mathrm{IFN}-\gamma^{+} \mathrm{IL}^{-} \mathrm{IL}^{-} 17 \mathrm{~A}^{-}\right)$in HT patients with severe hypothyroidism were significantly higher than those in mild patients. However, Th2 cells $\left(\mathrm{CD} 4^{+}\right.$IFN- $\gamma^{-}$IL- $4^{+}$IL-17A $\left.{ }^{-}\right)$were the opposite. The Th1/Th2 ratio of severe patients significantly increased, which suggested that the number and proportion of Th1/Th2 cells were related to the condition of HT, and Th1 cells were the main ones. Lin Li et al [12] found that the expression level of PBMC transcription factor GATA-3 in HT children was significantly lower than that in the control group, and the ratio of IFN- $\gamma / \mathrm{IL}-4$ was found in the direction of the polarization shift of Th1/Th2 cells in HT children. The levels of IFN- $\gamma$, T-bet/GATA-3, T-bet and free CD30 were significantly higher than those of the control group, while 
IL-4 significantly reduced, which suggested that the precursor $\mathrm{T}$ cells of children with $\mathrm{HT}$ had a tendency to differentiating and developing into Th1. Yang Yu et al. [13] found that the immune response of Th1 cytokines such as serum IL-2, IFN- $\gamma$ and so on in 10 children with HT was mainly shifted, and Th1/Th2 cell imbalance mainly shifted to Th1 cells, while children with GD shifted to Th2 cells and showed the advantage of $\mathrm{Th} 2$ cytokines. It suggested that Th1/Th2 cells and their cytokines played an important role in the pathogenesis of AITD. The abnormal expression of cytokines leaded to an imbalance in the immune network, which shifted the immune balance of Th1/Th2 cells, causing a series of pathological changes and immune dysfunction. It showed that the occurrence and development of AITD was intrinsically related to the imbalance of Th1/Th2 cells and the cytokines secreted by them. Shen Hao et al [14] found that the serum IL-2 of HT patients was significantly higher than that of the GD group and the control group by measuring the content of Th1 type cytokines IL-2 and Th2 type cytokines IL-5 in $45 \mathrm{GD}$ and 30 HT patients $(\mathrm{P}<0.05)$, but the difference between IL-5 and the control group was not statistically significant. IL-2 was significantly positively correlated with $\mathrm{TGAb}$ and thyroid microsomal antibody $(\mathrm{TM}-\mathrm{Ab})$ in HT patients $(\mathrm{P}<0.01)$, which suggested that Th1/Th2 cell immune balance was dominant in HT patients, while The GD patients are mainly Th2 cell immunization. The serum IL-5 of GD patients was significantly higher than that of HT group and control group $(\mathrm{P}<0.05)$, while the difference between IL-2 and control group was not statistically significant $(\mathrm{P}>0.05)$. Kuang Ruijuan [15] used 70 elderly AITD as the research object, among which 33 cases of HT patients had higher IL-2 levels, TM-Ab and TG-Ab than GD group and control group $(\mathrm{P}<0.05)$, while IL5 level was higher than HT group Significantly increased compared with the control group $(\mathrm{P}<0.05)$, which proved that Th1/Th2 cell imbalance was the main factor leading to the onset of AITD in the elderly. In HT, the imbalance of Th1/Th2 cells was biased towards the immune response dominated by Th1. Jiang Yanping [16] reported that the serum Th1/Th2 chemokines (CXCL10 and CCL22) levels and their ratios in 60 AITD patients with HT and GD were higher than those in the control group and healthy subjects $(\mathrm{P}<0.05)$. There was no statistically significant difference between the first-onset and the relapsed. Serum CXCL10 and CCL22 levels and their ratios were positively correlated with TPO-Ab, TG-Ab, $\mathrm{TR}-\mathrm{Ab}, \mathrm{CPR}, \mathrm{ESR}$ and thyroid volume, but were not correlated with FT3, FT4 and TSH. The results suggested that $\mathrm{Th} 1 / \mathrm{Th} 2$ jointly participated in the pathogenesis of AITD, but Th1 cells were the main ones. HT and GD patients had different Th1/Th2 shifts. GD was more significantly immune to Th2 immunity. After the treatment was improved, the balance of Th1/Th2 recovery in GD patients was more obvious than in HT patients. Wang Qi et al [17] conducted a retrospective analysis of 96 cases of AIT and found that Th1/Th2 and plasma TPO-Ab and TG-Ab levels in AIT patients were higher than those in non-inflammatory hyperthyroidism patients. The degree was $97.87 \%$, and there was no statistically significant difference in $\mathrm{Th} 1 / \mathrm{Th} 2$ and TR-Ab levels between the two groups $(\mathrm{P}>0.05)$. The levels of $\mathrm{TGAb}$ and TPOAb in the positive group were higher than those in the negative group $(\mathrm{P}<0.05)$. Th1/Th2 of AIT patients was significantly higher than that of non-inflammatory hyperthyroidism. TGAb, TPOAb and IgG4 levels were positively correlated $(\mathrm{P}<0.05)$. Th1/Th2, $\mathrm{TR}-\mathrm{Ab}$ and IgG4 levels were not correlated $(\mathrm{P}>0.05)$. It suggested that Th1/Th2 and plasma TPO-Ab, TG-Ab, TRAb levels were ideal indicators for the diagnosis of AIT, and the levels of TPOAb and TGAb were closely related to the progression of the disease. It showed that the shift of Th1/Th2 balance to Th1 could induce AIT, and the degree of proliferation and damage after infiltration could be completed by strengthening the expression of related cytokines. The degree of Th1/Th2 imbalance was closely related to the degree of AIT.

\section{Th17 Cells and Hashimoto's Thyroiditis}

Thl7 cells are a recently discovered subset of $\mathrm{CD} 4^{+} \mathrm{T}$ cells with different effector functions. They secrete IL-17A, IL-17F, IL-6, IL-21, IL-22, TNF- $\alpha$ and bind to CC Factor 6 (CCR6) and other cytokines, and interact with other cell subsets and cytokines. It also plays an important role in the inflammatory response and the pathogenesis of AID. With the deepening of the research on Th17 in the role of AID, the relationship among Th17 and its related factors with HT has attracted much attention. It has found that Th17 cells are involved in the development process of various AIDs such as rheumatoid arthritis, systemic lupus erythematosus, and inflammatory bowel disease [18]. Horie et al. believed that Th17 cells were a necessary condition for inducing AIT animal models, and the role of IL-17 and Th1 in the pathogenesis of HT also received attention. A research on the HT model of iodine-induced non-obese diabetes-H2h4 mice found that except for Th1 cells, the number of Th17 cells in thyroid and spleen increased significantly, and the TG-Ab titer of IL-17-deficient mice was significantly lower than wild mice. Animal model research on HT mice showed that Th1 could induce IL-17 knockout mice to develop HT, while Th17 induction alone couldnot induce IFN- $\gamma$ receptor knockout mice to induce HT [19]. The research on epidemiological found that the occurrence of HT was related to excess iodine. Thl7-induced immune response played an irreplaceable role in the pathogenesis of iodine-induced spontaneous animal model of HT. In the HT mouse model, it found that medium and high concentrations of iodine could promote the differentiation of naive $\mathrm{T}$ cells of mouse spleen to Th17 [20]. Th17 was a Th cell that induced autoimmunity, antagonizes Treg function, and had the characteristic of IL-23 dependent IL-17 production. IL-23 was not an essential factor for Thl7 cell differentiation, but had an important role in maintaining Th17 survival, phenotypic stability, and inducing Th17 development and maturity [21]. Shi et al. [22] 
believed that Thl7, not Thl, played a key role in the pathogenesis of HT. Wang et al [23] found that the number of Th17 cells in peripheral blood of HT patients increased. Bossowski et al [24] reported that the number of Th17 cells in peripheral blood of newly diagnosed children with HT increased significantly, which suggested that changes in the number of Th17 cells might affect the autoimmune function of children with HT. Xie Xiaoyan et al [25] found that the number of Th17 cells in peripheral blood of AITD patients increased significantly, especially in patients with hypothyroidism, which suggested that the increase in Th17 cells might be more closely related to hypothyroidism. Qin et al. [26] reported that the expression levels of IL-23, IL-17 mRNA and protein in thyroid tissue of HT patients significantly increased, and the two were positively correlated, while IFN- $\gamma$ was overexpressed, which suggested that Th17 cells, Th1 cells, IL 23 and IL-17 might play a synergistic role in the pathogenesis of HT and jointly participate in the occurrence and development of HT. IL-23 expressed at high levels in the thyroid tissue of patients with HT, and increased with the going up of IL-17A. The formation of the IL-17/IL-23 axis affected the progress of HT and played different roles in different stages of HT development [27]. Th17 cells in the thyroid tissue and peripheral blood of patients with AITD increased, and the ability of IL-6/IL-23 induced T cells to differentiate into Th17 cells was enhanced. Th17 cells, IL-17, and IL-22 in local thyroid tissue of HT patients were significantly higher than the control group. The expression of IL-17 in different glandular tissues was uneven, which was highly related to interstitial fibrosis, but not significantly related to lymphocyte infiltration [28], which indicated that the increase of IL-17 could aggravate the local inflammation of HT, promote the occurrence of fibrosis, and accelerate the progression of the disease [29]. Whether HT patients were light or heavy, Th17 cells significantly increased, indicating that Th17 and Th1 cells might play a synergistic role and jointly induce the development of HT. Serum IL-17 levels in HT patients significantly increased, and IL-17 and its induced inflammatory factors formed an inflammatory environment. By promoting the proliferation of inflammatory mediators and $\mathrm{T}$ cells, it was indirectly involved in the inflammatory response, resulting in the production of thyroid autoantibody and tissue damage. Figueroa-Vega et al [30] found that the serum IL-17 content of AITD (including GD and HT) patients was significantly higher than the healthy control group, which indicated that the ability of Th17 cells to produce IL-17 factor in HT patients was enhanced, especially in HT patients obvious. Li et al. [20] reported that the serum IL-17 level and IL-17 protein expression in local thyroid tissue of newly diagnosed HT patients were significantly higher than the control group, which suggested that IL-17 was closely related to the pathogenesis and course of HT. Kom et al. [31] found that the serum IL-17A peak appeared earlier than IFN- $\gamma$ in newly diagnosed HT patients, but the duration of IFN- $\gamma$ was longer than IL-17A, which suggested that Th17 cells and their cytokines might be early in HT played a role in the inflammatory response. Retinoic acid-related orphan receptor- $\gamma \mathrm{t}$ (Retinoidr-Elated Orphan Receptors (ROR- $\gamma \mathrm{t}$ ) was a key transcription factor for Thl7 differentiation. Yang et al. [32] found that IL-17, IL-23, IL-6 and Th17-related transcription factors ROR- $\gamma \mathrm{t}, \mathrm{ROR} \alpha$, and STAT3 in HT model mice all expressed at high levels. The expression of ROR- $\gamma \mathrm{t}$ and IL-17 gene and Th17 cells, IL-17 and IL-22 in thyroid tissue of HT patients increased, but there was no change in the control group. Xue Haibo et al [33] reported that the expression levels of Th17 cells and the secretion of IL-17 and ROR- $\gamma$ t in peripheral blood of HT patients increased, and the ratio of $\mathrm{Th} 17 / \mathrm{CD}^{+} \mathrm{T}$ cells was significantly positively correlated with the levels of serum TPOAb and TgAb. Chen Zijun [34] found that the levels of Th17 cells and IL-17 in AITD patients were higher than the normal control group, and the increase in HT was more significant. The increase of Th17 cells in HT patients was more obvious. Th17 cells were positively correlated with IL-17 levels, but not correlated with IL-6. Th17 cells and IL-17 levels in HT patients were positively correlated with TPO-Ab and TG-Ab, but not correlated with GD's TR-Ab. Chen Wanzhi [28] found that the levels of IL-17AmRNA and serum IL-17 in the thyroid tissue of HT patients were significantly higher than the control group, and the HT hypothyroidism group was higher than the normal hyperthyroidism group, while the Foxp3mRNA expression and IL-10 levels were significantly lower than the control group. There was no significant difference between the HT hypothyroidism group and the normal nail function group. It suggested that Th17 cell immune response in thyroid tissue and peripheral blood of HT patients was enhanced, and its changes might participate in the occurrence and development of HT. Zhao Jianyu [35] reported that Th17/CD4 ${ }^{+} \mathrm{T}$ cells in peripheral blood of GD and HT patients increased, especially in HT patients, so it can be used as one of the diagnostic basis of AITD, especially for the diagnosis of HT. Th17/CD4 ${ }^{+} \mathrm{T}$ cells in HT patients had a significant positive correlation with TG-Ab, but no significant correlation with TPO-Ab. Ruggeri et al. [36] reported that peripheral blood IL- $17^{+} \mathrm{CD} 4^{+} \mathrm{T}, \mathrm{IL}-22^{+} \mathrm{T}$ cells and serum IL-23, IL-22, IL-17A, and IFN- $\gamma$ of newly diagnosed HT patients were significantly higher than the control group. It currently believed that Foxp3 could regulate the expression level of Th17 cell transcription factor ROR- $\gamma$ t, thereby inhibiting the latter-mediated IL-17mRNA transcription and inhibiting the proinflammatory effect mediated by Th17 cells. Sun Qingkai et al [37] reported that plasma IL-6 concentration, $\mathrm{CD} 4^{+} \mathrm{IL}-17 \mathrm{~A}^{+} \mathrm{T}$ cell ratio, ROR- $\gamma \mathrm{t}$ expression level, $\mathrm{CD} 4^{+} \mathrm{T}$ cell phosphorylation signal transduction and transcriptional activator 3 (pSTAT3) expression in HT patients. And Thl7/Treg ratio were significantly higher than the control group $(\mathrm{P}<0.05)$, while $\mathrm{TGF} 0-\beta$ concentration, $\mathrm{CD}^{+} \mathrm{T}$ cell

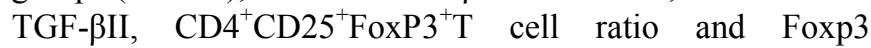
expression and Smad3, Smad4, TGF- Beta-induced early response gene 1 (TIEG1) and ItchmRNA expression were significantly reduced $(\mathrm{P}<0.05)$, and IL-6/TGF- $\beta$ was positively correlated with Thl7/Treg $(\mathrm{P}<0.05) . \quad \mathrm{CD}^{+} \mathrm{T}$ 
cytokine signal inhibitor 1 in HT patients' expressions of (SOCS1) and SOCS3 increased significantly $(\mathrm{P}<0.05)$, which was negatively correlated with Th17/Treg ratio $(\mathrm{P}<0.05)$. The $\mathrm{T} 4$ cells of peripheral blood $\mathrm{CD}^{+} \mathrm{T}$ cells stimulated by TGF- $\beta$ significantly increased, while Th17 cells and Thl7/Treg ratio significantly decreased $(\mathrm{P}<0.05)$. It suggested that abnormal IL-6/TGF- $\beta$ signal might be one of the factors that caused the imbalance of Thl7/Treg cells in HT patients. The above research results indicate that Th17 cells may be an important subset of T cells in the pathogenesis of HT. Th17 cells and their cytokines and transcription factors participate in the development of AITD, especially with HT.

\section{Treg Cells and Hashimoto's Thyroiditis}

Treg cells are a subset of $\mathrm{CD}^{+}{ }^{+} \mathrm{T}$ cells after $\mathrm{Th} 1$, Th2, and Th17. They can secrete cytokines such as IL-4, IL-10, and transform growth factor- $\beta$ (transforming growth factor, TGF- $\beta$ ), which directly or indirectly regulates the immune response, exerts an immunosuppressive effect, and plays an important role in inducing immune tolerance and immune homeostasis, thereby preventing the occurrence of AID by various mechanisms such as cytokines, intercellular contact, blocking $\mathrm{T}$ cell metabolism, cytolysis, and regulation of dendritic cell differentiation and function [38]. The highly expressed CD4 and CD25 molecules on the cell surface are important phenotypes of Treg. The research found that the decrease in the number of Treg cells and dysfunction in the peripheral blood of patients with AIT could cause hypothyroidism. Research on using transgenic mice found that although Treg might have nothing to do with immune tolerance, Treg (especially $\mathrm{CD} 4^{+} \mathrm{CD} 25^{+}$Treg) played a key role in HT hypothyroidism. The Foxp3 molecule expressed by the transcription factor Foxp3 was an important symbol of Treg. Foxp3 was a key transcription factor that maintains Treg development and function, and its expression depended on the presence of TGF- $\beta$. Horie et al [19] found that Thl could induce thyroiditis through autoimmune reaction. Due to the decrease of Treg cells and dysfunction in HT patients, Thl could not be well suppressed, resulting in Thl producing excessive amounts of IL- $\beta$, IFN- $\gamma$ and other cytokines, triggering a series of immune responses, thereby which caused thyroid tissue damage, resulting in thyroid Dysfunction induces hypothyroidism. The results of researches on Tregs cells in peripheral blood of HT patients were different. It had been reported in the literature that the number of peripheral blood Tregs cells, the transcription factor FoxP3 mRNA levels [33] and TGF- $\beta 1$ [39] levels in HT patients were lower than those in healthy controls. An important role of Treg was to suppress Thl-mediated autoimmunity and inflammatory response. Abnormal immune tolerance caused by decreasing Treg cell number or functional defect was related to the occurrence and severity of AIT [40]. ROR- $\gamma \mathrm{t}$ was an important transcription factor for Th17. Low-level Foxp3 in HT patients could not effectively suppress the expression of ROR-rt, and relatively low levels of TGF- $\beta$ could not effectively promote the development of Treg cells, which affected Treg's inhibition of Thl7 making Th17/Treg cell subsets in HT patients unbalanced and affecting thyroid function [41]. The dysfunction of Treg and Th17 cells played an important role in the pathogenesis of HT [42]. The immune imbalance of Treg/Th17 cells in peripheral blood of HT patients appeared in the normal stage of thyroid function, indicating that the immune imbalance of Treg/Th17 cell axis ran through the whole process of HT. Mao et al. [43] reported that the ratio of $\mathrm{CD}^{+} \mathrm{CD} 25^{+} \mathrm{Foxp} 3^{+}$ Treg cells $/ \mathrm{CD}^{+} \mathrm{T}$ cells in the peripheral blood of newly diagnosed AITD patients was significantly lower than GD and HT patients with normal thyroid function. In AITD patients, $\mathrm{CD} 4{ }^{+} \mathrm{CD} 25^{+}$Treg cells decreased, especially Foxp $3^{+} \mathrm{CD} 4^{+} \mathrm{CD} 25^{+}$Treg cells decreased, while activated $\mathrm{CD}^{+} \mathrm{T}$ cells increased. Guo Yuyu et al [44] found that the proportion of Treg cells in peripheral blood of HT patients $(0.026 \pm 0.013)$ was significantly lower than non-toxic goiter patients $(0.051 \pm 0.014, \mathrm{P}<0.01)$, which suggested that there was an imbalance of $\mathrm{T}$ cell subsets in HT patients. The significance decrease in the number of Treg may be involved in the pathogenesis of HT, which was consistent with the trend of Treg cells in other AID. Chen Wanzhi [28] found that the expression of Foxp3mRNA and IL-10 in thyroid tissue of HT patients was significantly lower than that of the control group, and there was no statistically significant difference between the HT hypothyroidism group and the normal group. Chen Wanzhi [28] found that the expression of Foxp3mRNA and IL-10 in thyroid tissue of HT patients was significantly lower than the control group, and there was no statistically significant difference between the HT hypothyroidism group and the normal group. Gao Shutao [45] et al. reported that the ratio of $\mathrm{CD} 4{ }^{+} \mathrm{CD} 25^{+} \mathrm{Tregs} / \mathrm{CD} 4^{+} \mathrm{T}$ cells in the peripheral blood PBMC of newly diagnosed HT patients, the inhibition rate of $\mathrm{CD} 4{ }^{+} \mathrm{CD} 25^{+}$Tregs on their own $\mathrm{CD} 4^{+} \mathrm{CD} 25^{-} \mathrm{T}$ cell proliferation, and the TGF- $\beta 1 \mathrm{mRNA}$ and Foxp3mRNA's expression level was significantly lower than the control group, and the $\mathrm{P}$ values were all inferior to 0.05 , but the number of $\mathrm{CD}^{+} \mathrm{T}$ cells in PBMC of newly diagnosed HT patients was not statistically different from the control group $(\mathrm{P}>0.05)$. It suggested that the $\mathrm{CD}^{+} \mathrm{CD} 25^{+}$ Tregs/CD4 ${ }^{+}$cell ratio, function and TGF- $\beta 1$, Foxp3mRNA expression levels in PBMC of HT patients significantly reduced. It also explained that the number and/or function of $\mathrm{CD} 4^{+} \mathrm{CD} 25^{+}$Tregs was involved in the development of HT. Yang Zhifang et al [46] found that the ratio of Treg/CD4 ${ }^{+} \mathrm{T}$ cells and Foxp3mRNA expression in $\mathrm{CD}^{+} \mathrm{T}$ cells in HT patients were significantly lower than the control group $(\mathrm{P}<0.01$ and $\mathrm{P}<0.05)$. There was no statistically significant difference in the proportion of $\mathrm{TregCD}^{+} \mathrm{T}$ cells of hypothyroidism, sub-hypothyroidism and normal thyroid function $(\mathrm{P}>0.05)$. Pearson correlation analysis showed that the number of Treg cells was negatively correlated with the patient's age and TPO-Ab level $(\mathrm{P}<0.05)$, which suggested that the number of Treg cells in peripheral blood of HT patients significantly reduced and accompanied by immune 
function defects, and was related to the patient's age and TPO- Ab level and was not related to thyroid function status. Bi Jianhua et al [47] found that the ratio of $\mathrm{CD} 4^{+} \mathrm{CD} 25^{+}$Foxp $3^{+}$Treg/CD $4^{+} \mathrm{T}, \quad \mathrm{CD} 4^{+} \mathrm{CD} 25^{+} \mathrm{Foxp}^{+}{ }^{+}$Treg/CD $4^{+} \mathrm{CD} 25^{+}$Treg and IL-10 in PBMC of HT patients were significantly lower than the control group $(\mathrm{P}<0.05)$. Dou Biao [48] reported that $\mathrm{CD} 4{ }^{+} \mathrm{CD} 25^{+}$Tregs $/ \mathrm{CD} 4^{+} \mathrm{T}$ cells in $\mathrm{HT}$ patients were lower than the control group $(\mathrm{P}<0.05) . \mathrm{C} 3$ and $\mathrm{C} 4$ levels were lower than those in Graves patients and the control group $(\mathrm{P}<0.05)$. The area under the curve (AUC) of the three indexes of $\mathrm{CD} 4{ }^{+} \mathrm{CD} 25^{+}$Tregs $/ \mathrm{CD} 4^{+} \mathrm{T}$ cells, $\mathrm{C} 3$ and $\mathrm{C} 4$ were $0.767,0.768$ and 0.736 respectively, and the AUC of the combined test was 0.923 , which was higher than that of the above three separate indexes $(\mathrm{P}<0.05)$. It suggested that the combined detection of $\mathrm{CD} 4{ }^{+} \mathrm{CD} 25^{+}$Tregs cells and complement $\mathrm{C} 3$ and $\mathrm{C} 4$ levels was helpful for the identification of HT and GD, and improved the accuracy of diagnosis. Most researches had shown that the number of Tregs cells in the peripheral blood of patients with HT reduced, but there were also reports of increasing and normal. For example, Fountoulakis et al [49] found that the number of Tregs cells in the peripheral blood of HT patients significantly increased, which may be related to the compensatory increase of Tregs cells of the body's immune system and suppression of the immune response of their own tissues. Marazuela et al. [50] reported that the number of Treg cells in the thyroid tissue and peripheral blood of AITD patients was significantly higher than the control group, and there was a functional defect, and their inhibitory ability significantly reduced. In peripheral blood of AITD patients, $T$ cells expressing Foxp3 and GITR increased, but the ability to inhibit cell proliferation decreased, and most of them could not down-regulate the autoimmune response and reduce the damage of inflammation to thyroid tissue [51]. Glick et al. [52] believed that there was no statistically significant difference among the number of Tregs cells in GD and HT patients and the control group, but in vitro experiments confirmed that the proliferation of Tregs cells inhibited the proliferation of effector $\mathrm{T}$ cells, which suggested that Treg cell function in AITD patients decreased. The above research results suggest that the role of Treg insufficiency in the development of AITD should be more important than the change in the number of Treg alone, which can reduce the body's ability to suppress the immune response of the body, leading to the occurrence of AITD. The immunosuppressive ability of Treg cells in thyroid tissue and peripheral blood of HT patients decreases, and the immune response of Th17 cells is enhanced. Treg/Th17 immune imbalance may participate in the development of HT, but its mechanism of action needs to be further study. In HT patients, the number of Treg cells decreases more frequently and is accompanied by dysfunction, but there are also reports of increased Treg numbers and no obvious changes. It may be related to the detection methods and signs, the number of cases and the degree of the disease. It needs to be prospectively studied in large samples.

\section{Outlook}

In conclusion, Th cells play a key role in regulating cellular immunity and humoral immunity. The imbalance of Th1/Th2 and Th17/Treg ratio is closely related to the pathogenesis of HT. Its cell number, ratio, immune activity, function and cytokines are abnormal. It leads to autoimmune dysfunction and plays an important role in the occurrence and development of HT. However, because of Th1/Th2 and Th17/Treg cells and their cytokines constituting a quite complex immune network system, the mutual influence and mechanism of action with each other are not completely clear. Strengthening research in this field will be of great significance to the early diagnosis and treatment of HT and the prevention of hypothyroidism, and to improve the quality of life of HT patients. By taking corresponding measures, through adjusting the $\mathrm{T}$ cell subsets and correcting the patient's immune imbalance, it is possible to open up new ways for the prevention and treatment of HT, and provide a new basis for the completely cure of HT and reduce the occurrence of hypothyroidism.

\section{Acknowledgements}

Cangzhou Key R \& D Plan and Guidance Project (Project No: 1833020011).

Natural Science Foundation of Cangzhou Medical College (No. 18Z015).

\section{References}

[1] Dumitru C, Kabat AM, Maloy KJ. Metabolic Adaptations of $\mathrm{CD} 4{ }^{+}$TCells in Inflammatory Disease [J]. Front Immunol, 2018, vol. 9, pp. 540 .

[2] Raphael I, Nalawade S, Eagar TN, et al. T cell subsets and their signature Cytokines inautoimmune and inflammatory disease [J]. Cytokine, 2015, vol. 74, pp. 5-17.

[3] Khanolkar A, Williams MA, Harty JT. Antigen experience shapes phenotype and function of memory Th1 cells [J]. PLoS One, 2013, vol. 8, pp. e65234.

[4] Sofian M, Aghakhani A, Farazi AA, et al. Serum profile of T helper 1 and $\mathrm{T}$ helper 2 cytokines in hepatitis $\mathrm{C}$ virus infected patients [J]. Hepat Mon, 2012, vol. 12, pp. e6156.

[5] Esfahanian F, Naimi E, Doroodgar F, et al. Th1/Th2 cytokines in patients with Graves'disease with or without ophthalmopathy [J]. Iran J Allergy Asthma Immunol, 2013, vol. 12, pp. 168-175.

[6] Hu S, Rayman MP. Multiple Nutritional Factors and the Risk of Hashimoto's Thyroiditis [J]. Thyroid. 2017, vol. 27, pp. 597-610.

[7] Xie YS, Qin WW. Migration of Thl/Th2 cytokines in autoimmune thyroid disease Internal Medicine. 2009, vol. 4, pp. 295-297.

[8] Ben-Skowronek I, Szewczyk L, Kulik-Rechberger B, et al. The differences in $\mathrm{T}$ and $\mathrm{B}$ cell subsets in thyroid of children with Graves' disease and Hashimoto's thyroiditis [J]. World J Pediatr. 2013, vol. 9, pp. 245-250. 
[9] Li LQ, Yang X, Feng J. Relationship Between Autoimmune Thyroid Disease and Cytokines of Th1/Th2. Medical Recapitulate, 2006, vol. 12, pp. 1160-1163.

[10] Jiang L, Wang CJ. Th1/th2 cells and autoimmune thyroid disease Anhui Med J, 2012, vol. 33, pp. 5629-5631.

[11] Nanba T. Watanabe $M$. Inoue $N$. et al. Increases of the Th1/Th2 cell ratio in severe Hashimoto's disease and in the proportion of Th17 cells in intractable Graves' disease [J]. Thyroid. 2009, vol. 19, pp. 495-501.

[12] Lin L, Xiong F, Zhao XD, et al. Th1/Th2 cells polarization in children with autoimmune thyroid diseases. J Clin Pediatr, 2009, vol. 27, pp. 1111-1114.

[13] Yang Y, Yang L, Xie LL, et al. Clinical significance of serum th1/th2 level in children with autoimmune thyroid disease Clin Focu, 2009, vol. 24, pp. 149-150.

[14] Shen $\mathrm{H}, \mathrm{Hu} \mathrm{L}$. The relationship between the imbalance of the Th1/Th2 cytokines and autoimmune thyroid disease. Chin J Prac Inter Med, 2009, vol. 29, pp. 253-253.

[15] Kuang RJ, Sun LH. The role and clinical value of th1th2 cell imbalance in elderly patients with autoimmune thyroid disease China \&Fore Med Treat, 2014, vol. 30, pp. 77-78.

[16] Jiang YP, Zhang HL. Preliminary Study of Th1/Th2 Imbalance in Different Types of Autoimmune Thyroid Disease. J Med Res, 2014, vol. 43, pp. 111-112.

[17] Wang Y, Kang ZQ, Zhang XF, et al. Changes and clinical significance of Th1/Th2cells and levels of plasma thyroid outoantibodies in patients with autoimmune thyroiditis. Prac $\mathrm{J}$ Clini Med, 2020, vol. 17, pp. 154-157.

[18] Hou ZJ, Mu ZX, Wang CC. Research Progress of Th17/Treg Cells and Their Transcription Factors in Autoimmune Diseases [J]. American Journal of Clinical and Experimental Medicine, 2019, vol. 7, pp. 83-92.

[19] Horie I, Abiru N, Sakamoto H, et al. Induction of autoimmune thyroiditis by depletion of $\mathrm{CD} 4{ }^{+} \mathrm{CD} 25^{+}$regulatory $\mathrm{T}$ cells in thyroiditis-resistant IL-17, but not interferon-gamma receptor, knock out nonobese diabetic-H2h4 mice [J]. Endocrinology, 2011, vol. 152, pp. 4448-4454.

[20] Li D, Cai W, Gu R, et al. Th17 cell plays a role in the pathogenesis of Hashimoto's thyroiditis in patients [J]. Clin Immunol, 2013, vol. 149, pp. 411-420.

[21] Toussirot E. The IL23/Th17 pathway as a therapeutic target in chronic inflammatory diseases [J]. Inflamm Allergy Drug Targets. 2012, vol. 11, pp. 159-168.

[22] Shi Y, Wang H, Su Z, et al. Differentiation imbalance of Th1/Th17 in peripheral blood mononuclear cells might contribute to pathogenesis of Hashimoto's thyroiditis [J]. Scand J Immunol. 2010, vol. 72, pp. 250-255.

[23] Wang S, Baidoo SE, Liu Y, et al. T cell-derived leptin contributes to increased frequency of $\mathrm{T}$ helper type 17 cells in female patients with Hashimoto's thyroiditis [J]. Clin Exp Immunol. 2013, vol. 171, pp. 63-68.

[24] Bossowski A, Moniuszko M, Idźkowska E, et al. Evaluation of $\mathrm{CD} 4{ }^{+} \mathrm{CD} 161^{+} \mathrm{CD} 196^{+}$and $\mathrm{CD} 4^{+} \mathrm{IL}-17^{+} \mathrm{Th} 17$ cells in the peripheral blood of young patients with Hashimoto's thyroiditis and Graves' disease [J]. Pediatr Endocrinol Diabetes Metab, 2012, vol. 18, pp. 89-95.
[25] XieXY, Xi' er NY, GuoT, et al. TheAlterationoftheTh1, Th2, Th17, Treg Cells in the Peripheral Blood in Patients with Grave' sDisease (GD) and Hashimoto' s Thyroiditis with Hypothyroidism. J Radioimmunology, 2011, vol. 24, pp. 290-293.

[26] Qin Q, Liu P, Liu L, et al. The increased but non-predominant expression of Th17-and Th1-specific cytokines in Hashimoto's thyroiditis but not in Graves' disease [J]. Braz J Med Biol Res [J]. 2012, vol. 45, pp. 1202-1208.

[27] Konca Degertekin C, Aktas Yilmaz B, Balos Toruner F, et al. Circulating Th17 cytokine levels are altered in Hashimoto's thyroiditis [J]. Cytokine, 2016, vol. 80, pp. 13-17.

[28] Chen WZ. Study on immune imbalance of Th17/Treg in patients with hashimoto's thyroiditis [D]. Master's degree thesis of nanchang university, 2012.

[29] Esfahanian F, Ghelich R, Rashidian H, et al. Increased Levels of Serum Interleukin-17 in Patients with Hashimoto's Thyroiditis [J]. Indian J Endocrinol Metab, 2017, vol. 21, pp. 551-554.

[30] Figueroa-Vega N, Alfonso-Pérez M, Benedicto I, et al. Increased circulating pro-inflammatory cytokines and Th17 lymphocytes in Hashimoto's thyroiditis [J]. J Clin Endocrinol Metab, 2010, vol. 95, pp. 953-962.

[31] Korn T, Reddy J, Gao W, et al. Myelin-specific regulatory T cells accumulate in the CNS but fail to control autoimmune inflammation [J]. Nat Med. 2007, vol. 13, pp. 423-431.

[32] Yang X, Gao T, Shi R, et al. Effect of iodine excess on Th1, Th2, Th17, and Treg cell subpopulations in the thyroid of NOD. H-2h4 mice [J]. Biol Trace Elem Res. 2014, vol. 159, pp. 288-296.

[33] Xue HB, Ma L, Li YB, et al. Correlation between Treg/Th17 cells imbalance and autoimmunity in Hashimoto thyroiditis Chin J Mode Med, 2012, vol. 22, pp. 67-71.

[34] Chen ZJ, Liu C, Li Q, et al. The change of Th17 lymphocytes and cytokines in autoimmune thyroid diseases Immu J, 2011, vol. 27 , pp. $785-788$.

[35] Zhao JH. Detection and significance of the alteration of Th17 cells in patients with Hashimoto's thyroiditis and Graves disease [D]. Jilin University Master's thesis, 2012.

[36] Ruggeri RM, Saitta S, Cristani M, et al. Serum interleukin-23 (IL-23) is increased in Hashimoto' s thyroiditis [J]. Endocr J, 2014, vol. 61, pp. 359-363.

[37] SunQK, Chen ZL, Li CH, et al. Investigation of theroles of interleukin 6 and transforming growth factor- $\beta$ signaling in Th17/Treg imbalance of Hashimotos thyroiditis. Chin J Endo Metab, 2015, vol. 31, pp. 320-326.

[38] Cheng AR, Cheng Y, Sun BL. Regulatory T Cells and Its Mechanism of Immunosuppression [J]. Chin J Clin Neurosci, 2014, vol. 22, pp. 438-444.

[39] Manolova I, Gerenova J, Ivanova M. Serum levels of transforming growth factor- $\beta 1$ (TGF- $\beta 1$ ) in patients with systemic lupus erythematosus and Hashimoto's thyroiditis [J]. Eur Cytokine Netw, 2013, vol. 24, pp. 69-74.

[40] Xue H, Wang W, Shan Z, et al. Dynamic changes of $\mathrm{CD} 4{ }^{+} \mathrm{CD} 25^{+}$regulatory $\mathrm{T}$ cells in NOD. $\mathrm{H}-2 \mathrm{~h} 4$ mice with iodine-induced autoimmune thyroiditis [J]. Biol Trace Elem Res. 2011, vol. 143, pp. 292-301. 
[41] Yoshimura A, Muto G. TGF- $\beta$ function in immune suppression [J]. Curr Top Microbiol Immunol. 2011, vol. 350, pp. $127-47$.

[42] Roberto González-Amaro, Mónica Marazuela. T Regulatory (Treg) and T Helper 17 (Th17) Lymphocytes in Thyroid Autoimmunity. Endocrine, 2016, vol. 52, pp. 30-38.

[43] Mao C, Wang S, Xiao Y, et al. Impairment of regulatory capacity of $\mathrm{CD} 4{ }^{+} \mathrm{CD} 25^{+}$regulatory $\mathrm{T}$ cells mediated by dendritic cell polarization and hyperthyroidism in Graves' disease [J]. J Immunol. 2011, vol. 186, pp. 4734-4743.

[44] Guo YY, Lu YL, Zhai HL, et al. Analysis of peripheral blood regulatory $\mathrm{T}$ cell count and clinical significance in patients with Hashimoto's thyroiditis. Clin Focu, 2011, vol. 26, pp. 62-64.

[45] Gao ST, Zhu TN, Zhang YN, et al. The frequency and function of $\mathrm{CD}^{+} \mathrm{CD} 25^{+}$regulatory $\mathrm{T}$ cells in autoimmune thyroid diseases Chin J Immu, 2011, vol. 27, pp. 269-273.

[46] Yang ZF, Wu F, Tang JZ, et al Study about the Regulatory T Cells in Patients with Hashimoto's Thyroiditis Chin Gene Prac, 2013, vol. 16, pp. 2477-2479.
[47] Bi JH, Hao LX, Huang F, et al. Study on the clinical significance of detection of peripheral blood $\mathrm{CD} 4{ }^{+} \mathrm{CD} 25^{+}$Foxp $3^{+}$regulatory $\mathrm{T}$ cells and IL-10 in patients with Hashimoto thyroiditis. J Clin Exper Med, 2017, vol. 16, pp. 38-40.

[48] Dou B. Significance of combined detection with $\mathrm{CD} 4{ }^{+} \mathrm{CD} 25^{+}$regulatory $\mathrm{T}$ cells and complement $\mathrm{C} 3, \mathrm{C} 4$ levels for early diagnosis of HT and Graves disease. J Medical Forum 2019, vol. 40, pp. 24-27.

[49] Fountoulakis S, Vartholomatos G, Kolaitis N, et al. HLADR expressing peripheral $\mathrm{T}$ regulatory cells in newly diagnosed patients with different forms of autoimmune thyroid disease [J]. Thyroid, 2008, vol. 18, pp. 1195-1200.

[50] Marazuela M, García-López MA, Figueroa-Vega N, et al. Regulatory $\mathrm{T}$ cells in human autoimmune thyroid disease [J]. J Clin Endocrinol Metab. 2006, vol. 91, pp. 3639-3646.

[51] Bao QY, Xu YS, Yang HY. Regulatory T Cells and Endocrine Diseases. Med Reca, 2014, vol. 20, pp. 3278-3280.

[52] Glick AB, Wodzinski A, Fu P, et al. Impairment of regulatory T-cell function in autoimmune thyroid disease [J]. Thyroid, 2013, vol. 23, pp. 871-878. 